\title{
ROSINA (RSI) is part of a CACTA transposable element, TamRSI, and links flower development to transposon activity
}

\author{
Mario Roccaro • Yubin Li · Hans Sommer • \\ Heinz Saedler
}

Received: 12 December 2006 / Accepted: 2 May 2007 / Published online: 23 June 2007

(C) Springer-Verlag 2007

\begin{abstract}
ROSINA (RSI) was isolated as a DNA binding factor able to bind to the CArG-box present in the promoter of the MADS-box gene DEFICIENS of Antirrhinum majus. The mosaic nature of $R S I$ and its multi-copy presence in the A. majus genome indicated that $R S I$ could be a part of a mobile genetic element. Here we show that $R S I$ is a part of a CACTA transposable element system of A. majus, named TamRSI, which has evolved and is still evolving within the terminal inverted repeats (TIRs) of this CACTA transposon. Interestingly, $R S I$ is always found in opposite orientation with respect to the transcription of a second gene present within the CACTA transposon, which encodes a putative TRANSPOSASE (TNP). This structural configuration has not yet been described for any member of the CACTA transposons superfamily. Internal deletion derivatives of the TamRSI produce aberrant RSI transcripts (RSI$A T S$ ) that carry parts of the RSI RNA fused to parts of the TNP RNA. In addition, an intriguing seed phenotype shown by RNAi transgenic lines generated to silence $R S I$, relate TamRSI to epigenetic mechanisms and associate the control of flower development to transposon activity.
\end{abstract}

Communicated by M.-A. Grandbastien.

Electronic supplementary material The online version of this article (doi:10.1007/s00438-007-0245-x) contains supplementary material, which is available to authorized users.

M. Roccaro $(\square) \cdot$ H. Sommer $\cdot$ H. Saedler

Max-Planck-Institut für Züchtungsforschung,

Carl-von-Linne'-Weg 10, 50829 Koeln, Germany

e-mail: roccaro@mpiz-koeln.mpg.de

Present Address:

Y. Li

Waksman Institute, Rutgers University,

190 Frelinghuysen Road, Piscataway, NJ 08854-8020, USA
Keywords Transposable elements · dsRNA · Imprinted genes $\cdot$ Antirrhinum majus

\section{Introduction}

Transposable elements (TEs) or transposons are DNA sequences capable to change their position within a genome (McClintock 1950). TEs, genetically defined by Barbara McClintock (1950), were regarded with scepticism for a long period because genes and even chromosomes were envisioned as static strings of genetic material with little freedom for structural rearrangements. McClintock named them "controlling elements", thus suggesting a role in the regulation of gene activity (McClintock 1956). Their potential regulatory function was overshadowed by the operon theory (Jacob and Monod 1961) and even after TEs had regained scientific interest (Nevers and Saedler 1977), some scientists regarded them as "selfish" DNA with no role in cellular processes beside their own survival (Doolittle and Sapienza 1980). A prototype of DNA transposons is represented by the $E n / S p m$ element of maize, which belongs to the large family of the CACTA TEs. Several structural features, some in common with other types of TE families, characterise the CACTA family, to which the Taml element of Antirrhinum majus also belongs. Firstly, at both ends of a CACTA transposon, terminal inverted repeats (TIRs) are found. Secondly, transposons induce characteristic target site duplications upon insertion (Bonas et al. 1984; Schwarz-Sommer et al. 1984). In addition, sequence repeats are also found at sub-terminal positions with respect to the TIRs (Kunze and Weil 2002). The integrity of the TIRs and of the sub-terminal repeats is essential for the transposition (Schiefelbein et al. 1988). En/Spm codes for at least two proteins with separate functions that are produced 
from a single precursor transcript by alternative splicing (Pereira et al. 1986; Masson et al. 1989). One protein, named TNPD, represents the putative transposase required for the excision/integration process during transposition. The second protein, named TNPA, is a factor with multiple functions, some of them reflecting its ability to bind DNA (Gierl et al. 1988; Trentmann et al. 1993). Ultimately, the TNPA can act as a positive and negative regulator of its own activity (McClintock 1965). This function is complex and involves both an auto-regulatory mechanism and DNA methylation "states" of a GC-rich region within the element (Fedoroff et al. 1995). Very little is known how the methylation "states" (epigenetic states) of these cis-regulatory regions are established. More recently, a new link between transposon activity and epigenetic phenomena such as paramutation, imprinting and gene silencing has been established in plants as well as in animals (Ketting et al. 1999; Tabara et al. 1999; Lippman et al. 2004; Martienssen et al. 2004; Alleman et al. 2006).

ROSINA (RSI) was isolated as a factor able to bind a specific promoter region of the DEFICIENS gene and to modulate petal and stamen development (Roccaro et al. 2005). The RSI gene is present in multiple copies within the genome of Antirrhinum majus. RSI encodes a product that shows weak similarity to several $\mathrm{En} / \mathrm{Spm}$-like proteins from different plant species. These findings suggest that the RSI gene might be part of a transposable element. Here, the genomic organization of several RSI copies is described, demonstrating that $R S I$ is indeed a part of a CACTA transposable element. Sequence analysis shows that $R S I$ is found in opposite orientation to a putative TRANSPOSASE (TNP) carried by the transposon. CACTA transposons encoding two genes with a convergent direction of transcription have not been yet described. In addition, internal deletion derivatives of TamRSI fuse parts of the RSI gene with the TNP. These types of TamRSI copies are able to produce aberrant transcripts (RSI-ATs) that carry parts of RSI in antisense orientation. The identification of double-stranded RNA (dsRNA) formed by sense and antisense RSI transcripts, and a parent-of-origin effect on the seeds revealed by backcrosses of RSI-RNAi transgenic lines with wild type lines, relate RSI to epigenetic mechanisms of gene regulation, and associate the control of flower development to transposon activity.

\section{Materials and methods}

Plant material and growth conditions

All inbred lines of A. majus were grown in the greenhouse at $18-25^{\circ} \mathrm{C}$ under $16 \mathrm{~h}$ light and $8 \mathrm{~h}$ dark. Seeds were germinated on the surface of moist vermiculite at $20^{\circ} \mathrm{C}$ under the same light conditions. For outcrossing, immature anthers were removed from the female parent when the flower was still closed. Seeds were collected as soon as the fruit began to dehisce. The mutant lines opulentiflora and pleiocotiledona were obtained from the A. majus mutant collection maintained at the IPK, Gaterleben (Germany). The two RNAi lines were described previously (Roccaro et al. 2005). The weight of the seeds of each genotype was determined by randomly selecting 40 seeds from three different capsules. The average weight of a seed was obtained dividing by 40 .

\section{DNA extraction and Southern blot hybridization}

DNA extractions were performed using the method of Coen et al. (1986). Southern blots were performed as described in Sambrook et al. (1989). For high stringent conditions of hybridisation, Hybond-N filters containing genomic DNA were incubated overnight at $65^{\circ} \mathrm{C}$ in 50 or $100 \mathrm{ml}$ of $3 \mathrm{X}$ SSC, $0.1 \%$ SDS, $0.2 \%$ ficoll and $0.2 \%$ polyvinylpyrrolidone with the addition of salmon-sperm DNA (end concentration $0.1 \mathrm{mg} \mathrm{ml}^{-1}$ ). After hybridisation, the filters were washed twice in $0.5 \mathrm{X} \mathrm{SSC}, 0.1 \%$ SDS for $10-15 \mathrm{~min}$ at the same temperature used for hybridisation. For low stringent conditions of hybridisation the filters were incubated overnight at $50^{\circ} \mathrm{C}$ in 50 or $100 \mathrm{ml}$ of $5 \mathrm{X} \mathrm{SSC}, 0.1 \%$ SDS, $0.2 \%$ ficoll and $0.2 \%$ polyvinylpyrrolidone with the addition of salmon-sperm DNA. After hybridisation, the filters were washed twice in $3 \mathrm{X} \mathrm{SSC}, 0.1 \%$ SDS for $10-15 \mathrm{~min}$ at the same temperature as for hybridisation. Southern blots of PCR or RT-PCR products were performed using alkaline blot. Filters were hybridised in $3 x$ SSC $0.1 \%$ SDS at $70^{\circ} \mathrm{C}$ for $6 \mathrm{~h}$. Filters were washed twice with $0.1 \mathrm{x}$ SSC $0.1 \%$ SDS for 30 min each time.

\section{Genome walk}

Genome walk was performed following the strategy used in the GENOMEWALKER ${ }^{\mathrm{TM}}$ kit (CLONTECH) as indicated in the manufacturer's instructions with minor modifications. Briefly, $2.5 \mu \mathrm{g}$ of A. majus genomic DNA was independently digested with several blunt end restriction endonucleases. After a phenol/chloroform extraction and ethanol precipitation, the digested DNA samples were subjected to an adaptor ligation. The adaptor is made by two partially complementary oligonucleotides, one of which (the lower strand) is phosphorylated at its $5^{\prime}$ end whereas its $3^{\prime}$ end carries an amino group. This amino group blocks extension by Taq polymerase at the $3^{\prime}$ end of the adaptorligated genomic fragments preventing the amplification of unspecific PCR products primed with the adaptor primer, but favouring the amplification of PCR products primed with the gene-specific primer and the adaptor primer. The 
digested genomic DNA was ligated in the presence of $2.0 \%$ PEG 8000 at $16^{\circ} \mathrm{C}$ overnight with an excess of adaptor in $50 \mu 1$ reaction volume. The ligated DNA was diluted to $50 \mathrm{ng} / \mu \mathrm{l}$ and $1 \mu \mathrm{l}$ used in the first nested PCR reactions. Only 15 cycles of amplification were performed in the first nested PCR, consisting of a denaturing step at $95^{\circ} \mathrm{C}$ for $20 \mathrm{~s}$, the annealing step at $65^{\circ} \mathrm{C}$ for $1 \mathrm{~min}$ and the extension step at $68^{\circ} \mathrm{C}$ for $4 \mathrm{~min}$ and $30 \mathrm{~s}$. One microliter of this first nested PCR reaction was used for the following nested reaction, using 25 cycles of amplification with the same time and temperature conditions. Finally, $5 \mu \mathrm{l}$ of last nested PCR reaction were loaded on $0.7 \%$ agarose gel to resolve the PCR products, which was alkaline-blotted and hybridised with a specific TamRSI flanking region as probe. The DNA of the hybridising bands were subsequently isolated and cloned using $\mathrm{TOPO}^{\mathrm{TM}}$ blunt end vector (Invitrogen).

\section{RT-PCR, PCR amplifications, and double-stranded RNA} detection

The RT-PCRs were performed as described previously (Roccaro et al. 2005). PCR amplification of products longer than $4.5 \mathrm{~kb}$ were amplified with the Expand High Fidelity system (ROCHE), using amplification programs as indicated in the manufacturer's instructions. Doublestranded RNA was detected according to the protocol described in Korneev (1999). The list of the primers used in this study is found in supplementary Materials and methods, Table 1 .

\section{Results}

RSI is a multicopy gene

Southern blot hybridization allows estimation of the copy number for a gene in a given genome when highly stringent conditions are used. Using RSI full-length cDNA as a probe, Southern blot analysis revealed a relatively high number of hybridizing bands, depending on the restriction enzyme and on which A. majus lines were used (Fig. 1). Approximately 12-14 RSI copies were estimated per A. majus genome. Within the A. majus species some bands were polymorphic (Fig. 1).

The copy number also changed within the Antirrhinum family, with A. siculum showing the lowest number of copies (Suppl. Fig. 1). Hybridizations at low stringent conditions showed that the RSI sequence is conserved only within the Antirrhinum family and in phylogenetically closely related species such as Misopates orontium (highest copy number), but is absent in more distant relatives of $A$. majus like Linaria, and in unrelated species, including the monocot barley (Suppl. Fig. 1).
Screening of a genomic library with the RSI cDNA as a probe yielded 43 recombinant phages, which were assigned to distinct groups. Sequence comparison among the unique clones showed a high degree of similarity with each other over most of their length (similarity higher than 93\%), but with an abrupt sequence change at their $5^{\prime}$ end of the RSI gene. At the divergence point of each genomic clone a CACTA terminal inverted repeat (TIR) characteristic of the $\mathrm{En} / \mathrm{Spm}$ super-family of transposable elements was found (Bonas et al. 1984; Pereira et al. 1986). Beside the $5^{\prime}$ end CACTA signature, none of the genomic clones carried a CACTA TIR at the $3^{\prime}$ end or contained other structural similarities with any of the known CACTA type of TEs, such as $5^{\prime}$ or $3^{\prime}$ sub-terminal repeats and/or conserved transposase motifs. Nevertheless, the weak similarity of RSI with transposon like-proteins, its multi copy presence in the $A$. majus genome and in a phylogenetically closely related species, together with the CACTA signature at the $5^{\prime}$ end of all analysed copies, suggested that $R S I$ could be part of a TE of the CACTA super-family. Therefore, hallmarks required to associate $R S I$ to a transposon were systematically investigated such as TIRs, ability to transpose and/or to generate deletion derivatives.

The RSI gene is located between TIRs of an En/Spm-like transposable element

DNA transposable elements are structurally characterised by the presence of terminal inverted repeats (TIRs) and are flanked, at the site of insertion, by target site duplications (TSD). Additional genomic library screens using $c R S I$ as probe did not lead to the identification of an RSI clone containing a $3^{\prime}$ end CACTA TIR. Therefore, a different approach was used to verify the presence of TIRs and TSD. This approach takes advantage of the strategy used to clone Taml (Bonas et al. 1984) and it represents an unequivocal way to verify the presence of the TIRs. This approach consisted of the identification of a polymorphism in segregating F2 populations of several A. majus lines, using the various regions flanking the $5^{\prime}$ end CACTA signature as probes. A polymorphism would indicate the absence and/or a rearrangement of the transposon at a specific locus that could deliver the $3^{\prime}$ end flanking sequence with respect to the transposon insertion and/or the other transposon end. In the case of the absence of the transposon from the investigated locus, the $3^{\prime}$ end flanking sequence can in turn be used as a probe to clone the $3^{\prime}$ end CACTA TIR from the line carrying the transposon (Suppl. Fig. 2). Two of these flanking regions, belonging to two unique $R S I$ genomic clones (RSI-2 and RSI-3) detected two polymorphisms, respectively (Suppl. Fig. 2, probe 1 and probe 3). The polymorphic bands were cloned. Both the $165 E$ segregating bands contained part of the $R S I$ gene $5^{\prime}$ end and further upstream regions. 
Fig. 1 Estimation of the RSI copy number by Southern blot hybridization. Antirrhinum majus genomic DNA from the wild type lines $165 E$ and Sippe 50 was digested with different restriction endonucleases and hybridized with full length $R S I$ cDNA as a probe. Polymorphisms were detected between the two lines. The lane $M$ indicates the DNA markers with the corresponding size values

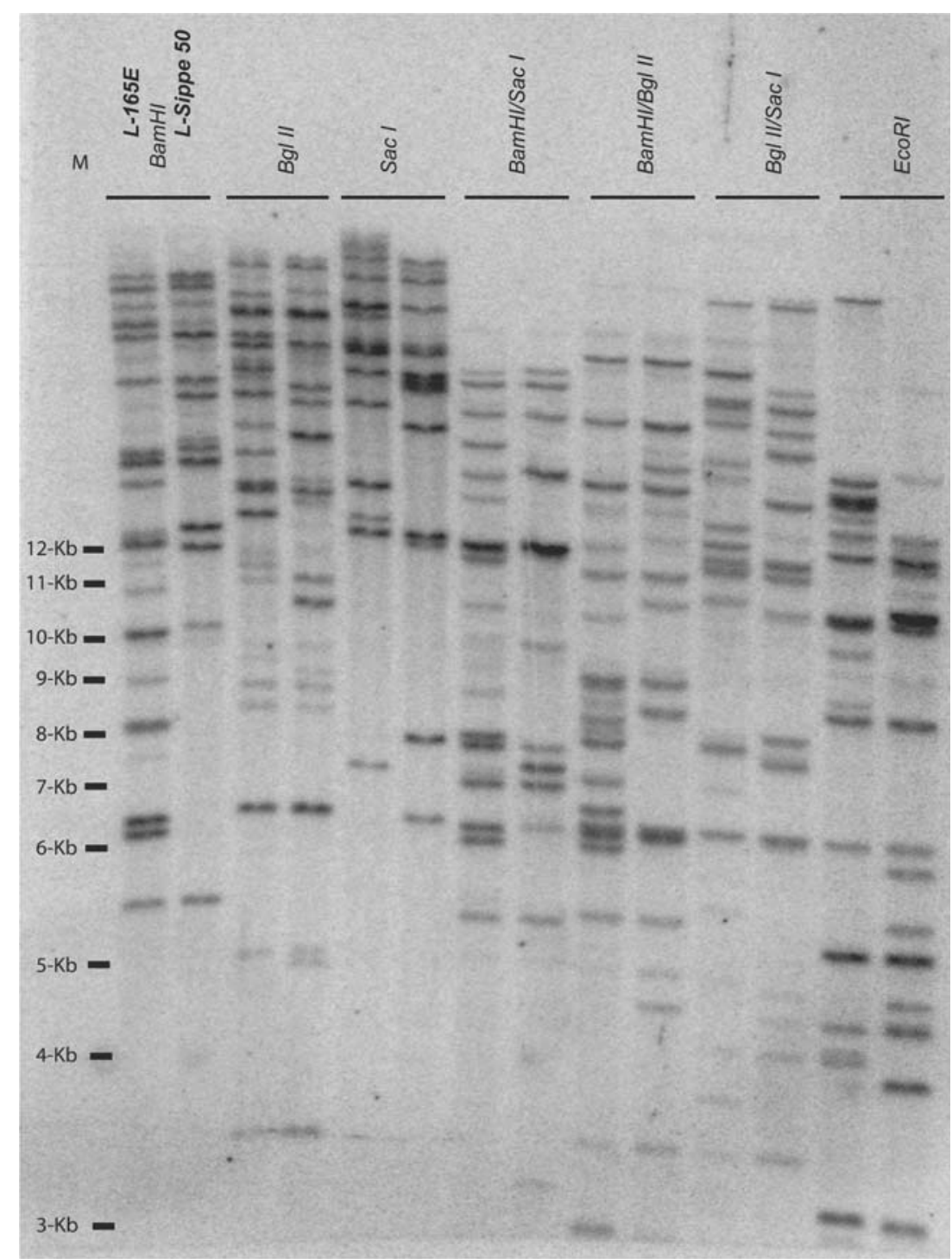

The despenteado-1 and -2 (desp-1, desp-2) (Wilkinson et al. 2000) segregating fragments carried the same RSI $5^{\prime}$ end flanking regions and at their $3^{\prime}$ end new sequences, but no RSI parts. Next, we hybridised restricted DNA of the segregating F2 population of desp-1 with the newly isolated 3' end sequence as a probe (Suppl. Fig. 2, probe 2). A new polymorphism was found: one band of about $5 \mathrm{~kb}$ belonging to the desp- 1 line and a new band of about $9.5 \mathrm{~kb}$ belonging to the $165 E$ line (Suppl. Fig. 2). Attempts to clone the $9.5 \mathrm{~kb}$ band from the EcoRI digest as well as polymorphic bands arising from other restriction endonuclease digests failed, suggesting that the $3^{\prime}$ end $R S I$ sequence could be deleterious or not stable in the phages and/or in E. coli strains used to clone and amplify the polymorphic genomic DNA fragment. In addition, attempts to span by PCR the complete RSI-2 and RSI-3 loci using primer combinations on the corresponding $5^{\prime}$ and $3^{\prime}$ end flanking sequences failed. By Southern blot hybridisation a minimal length of $21 \mathrm{~kb}$ was calculated for the $R S I-2$ copy (data not shown; a partial genomic structure of RSI-2 is shown in Suppl Fig. 3). Although it was not possible to clone the new polymorphic DNA fragment from the line $165 E$, the $3^{\prime}$ end flaking regions of RSI-2 and RSI-3 provided two new unique sequences where to anchor PCR primers to verify, using the genome walk strategy, the presence of a CACTA TIR at the $3^{\prime}$ end of these two RSI copies.

Genome walk and identification of the TIRs and TSD

The genome walk strategy (see Materials and methods) allows walking towards an unknown genomic sequence from a known genomic sequence, in our case from the new identified $3^{\prime}$ end flanking regions. A genome walk from the known $3^{\prime}$ end $R S I$ sequence was prohibitive due to the high degree of sequence identity among the various copies. This strategy was successful and it delivered sequences carrying a CACTA signature in the correct orientation with respect 
to the $5^{\prime}$ end CACTA signature of RSI-2 and RSI-3, respectively. In addition, the RSI-2 contained an imperfect target site duplication (TSD) TCA/TC, whereas the RSI-3 copy contained a perfect 3 bp TDS ATA/ATA flanking the insertion site. This finding supported the idea that the RSI-2 and RSI-3 copies were located within the TIRs of a CACTA transposon. Hence, these RSI copies represented new insertions in the $165 E$ line because the TSDs were not found in the lines desp-1 and desp-2. Although, we could not isolate the complete RSI-2 and RSI-3 genomic copies, primers designed on the TIRs of these two copies allowed us to amplify by PCR a product of about $10 \mathrm{~kb}$ containing the full $R S I$ coding region and representing a new $R S I$ copy (RSI-6) (Fig. 2). Sequence analysis of RSI-6 demonstrated that a putative TRANSPOSASE (TNP) gene was present within the CACTA TIRs in opposite orientation of RSI (Fig. 2). This type of transposon structure has not yet been described for any member of the En/Spm family of DNA transposons, and hence represents a novelty. According to the nomenclature of the A. majus transposons (Bonas et al. 1984), this new type of transposon was named TamRSI followed by a number to indicate the corresponding specific copy.

Mapping and excision analysis of TamRSI

Characteristic for mobile TEs is their localisation at nonallelic positions and their ability to transpose from one location to another within a genome leaving behind, in some cases, footprints at the site of excision (Sommer et al. 1988). To further understand the nature of TamRSI, dominant and co-dominant markers were selected to map some copies using an A. majus X A. molle F2 population (Schwarz-Sommer et al. 2003). Two copies, TamRSI-3 and a later isolated TamRSI-4, mapped to linkage group 6 (Lg6) and $\mathrm{Lg} 5$ respectively (data not shown). Therefore, it appeared that TamRSI-2 and TamRSI-3 were located in the Lg6 because of their linkage with the DESP locus. The mapping experiment revealed the non-allelic positions of the TamRSI copies.

Another diagnostic feature of TEs is the generation of DNA footprints upon excision. Since it was not possible to cover the entire locus of TamRSI-2 or TamRSI-3 by PCR, amplification of products able to hybridise with probes flanking these two copies would represent somatic and/or germinal excision events. Primer pairs specific to the flanking sequences of TamRSI-2 and TamRSI-3 copies, respectively, were used in a series of nested PCR amplifications to detect excision events. As shown in Fig. 3, TamRSI-2 was able to excise from its genomic location. The excision events were imperfect, generating footprints (deletions) (Fig. 3). Similarly, the movement of the TamRSI-3 copy was detected (Suppl. Fig. 4). These experiments clearly demonstrated that TamRSI could transpose and integrate at non-allelic positions, two diagnostic features for mobile DNA transposons.
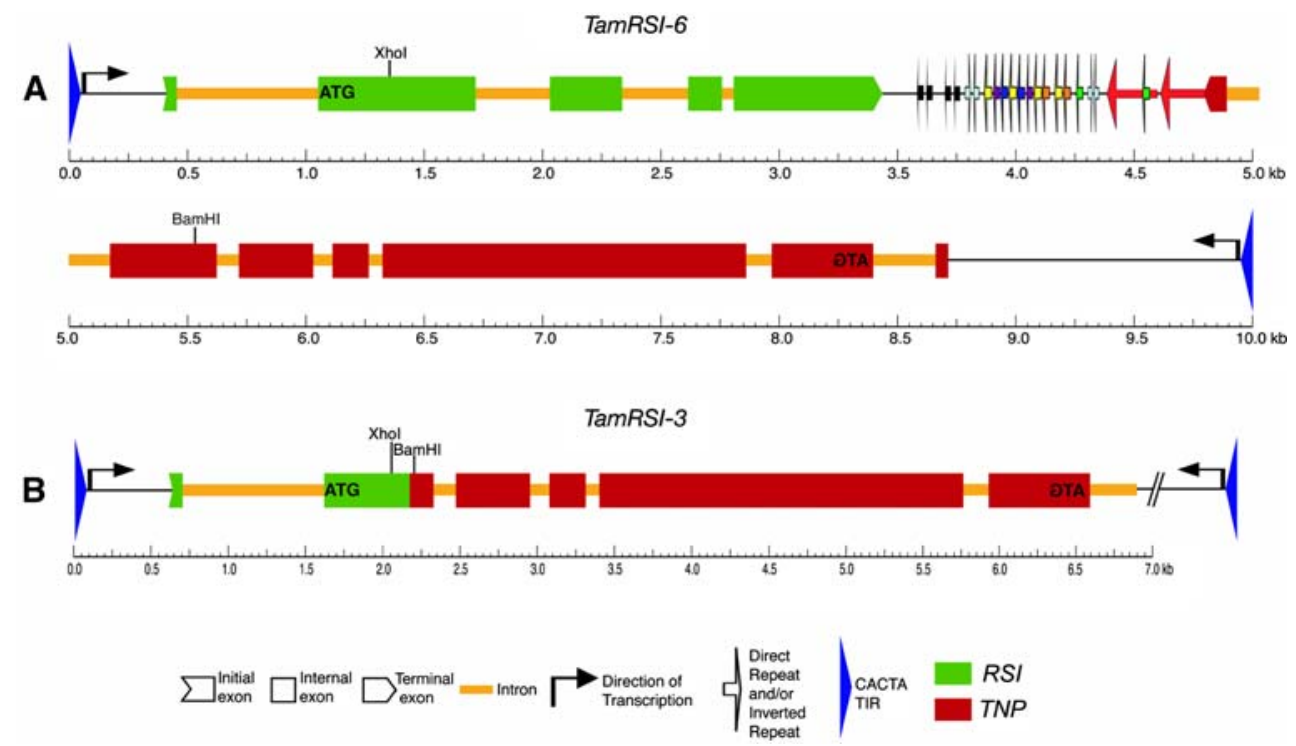

Fig. 2 Schematic representation of the $10 \mathrm{~kb}$ long genomic structure of TamRSI-6 (AM498638) (a) and of the partial TamRSI-3 structure (AM502588 and AM 502589) (b). Unlike the RSI genomic structure of TamRSI-6, which was derived by sequence comparison of the $10 \mathrm{~kb}$ genomic PCR product with cDNA clones, the genomic structure of TNP was predicted using GENSCAN (http://www.genes.mit.edu/
oldGENSCAN.html) and checked manually for exon/intron borders. The RSI gene consists of five exons and four introns, with the first intron in the $5^{\prime}$ UTR directly adjacent to the first putative RSI translational start codon (ATG). In $\mathbf{b}$, the deletio-fusion event in TamRSI-3 occurred between the XhoI and BamHI sites. The TamRSI-3 structure is not complete as indicated by the two slash lines 
A

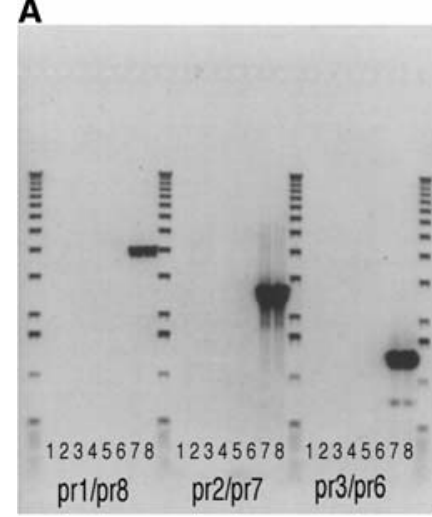

B

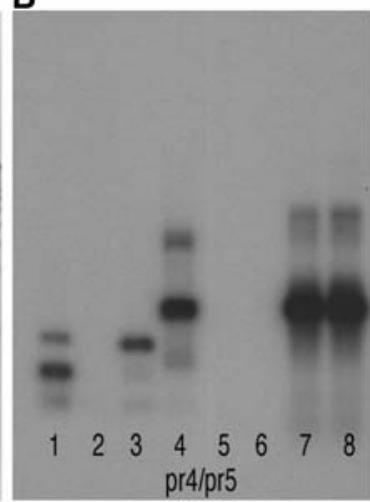

C

TamRSI2

line $165 E$

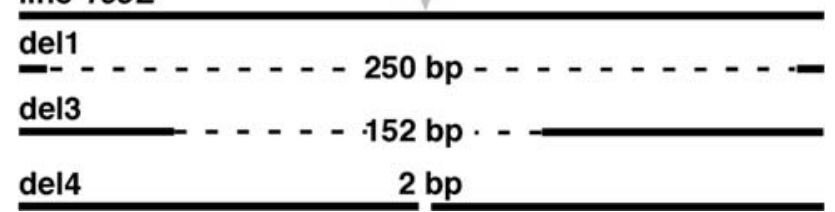

Fig. 3 Detection of TamRSI-2 transposition $(\mathbf{a}, \mathbf{b})$ and three examples of footprints detected after its excision (c). Genomic DNA from four pools was used, with each pool containing twenty-five $165 \mathrm{E}$ plants (samples 1, 2, 3 and 4). Two "negative" controls (presence of TamRSI2 at the investigated locus: no PCR product amplified) represented by DNA extracted from a single individual plant of $165 E$ (sample 5) and Sippe 50 (sample 6) lines, respectively, and two positive controls (absence of TamRSI-2 from the investigated locus: PCR product amplified) represented by DNA extracted from Line 91 (sample 7) and from Pallida-recurens (sample 8) respectively, were also included. a Agarose gel electrophoresis of the first three nested PCR amplifications and b Southern blot hybridization of amplified PCR products from the last primer combination. The $5^{\prime}$ end flanking region of TamRSI-2 was used as a probe. $\mathbf{c}$ The three strongest hybridizing bands (sample 1, 3 and 4 in B) were cloned and sequenced to show the type of excision events (deletion: del-1, del-3 and del-4) of TamRSI-2

\section{Deletion derivatives as source of aberrant $R S I$ transcripts}

Internal deletions within the TEs are common and are generated by aberrant transposition events (Kunze and Weil 2002). The known portion of the TamRSI-3 copy has a genomic structure that fuses part of the TNP coding region with the $5^{\prime}$ end of the RSI coding region (Fig. 2). Primer pairs designed to detect variants of internal deletions similar to TamRSI-3 amplified several genomic PCR products of different lengths ranging from 2.1 to $6.5 \mathrm{~kb}$ depending on the plant line used. Some of these products were able to hybridise with both a TNP and with a RSI probe (Suppl. Fig. 5). Transposon structures like TamRSI-3 (Fig. 2) have the potential to produce aberrant RNA molecules. We have previously reported the isolation of an aberrant $R S I$ transcript called RSI-ATI (Roccaro et al. 2005). The identification of other internal deletion variants of TamRSI (Suppl. Fig. 5) suggested the existence of additional aberrant RSI transcripts (RSI-ATs), similar to RSI-ATI, formed by parts of the RSI fused to parts of the TNP. To identify these predicted RSI-ATs, RT-PCR experiments were carried out in different A. majus lines. Figure 4 demonstrates the presence of additional RSI-ATs. Some of these transcripts are expressed in a specific manner (Fig. 4: 165E vegetative meristems). In addition, in some lines, these transcripts seemed to be suppressed or reduced to a low level not detectable under these conditions (Fig. 4).

The presence of these aberrant transcripts indicated a complex transcriptional regulation among the TamRSI copies. Since they carry antisense sequences of the RSI coding region, they have the potential to form RNA duplexes with the full-length $R S I$ transcripts, which in turn can regulate transcription of the TamRSI copies via post-transcriptional gene silencing (PTGS) (Tijsterman et al. 2002 and references therein), and/or via transcriptional gene silencing (TGS) (Matzke et al. 2001). Thus, the formation of RNA duplexes and their possible regulatory function were investigated as described in the following section.

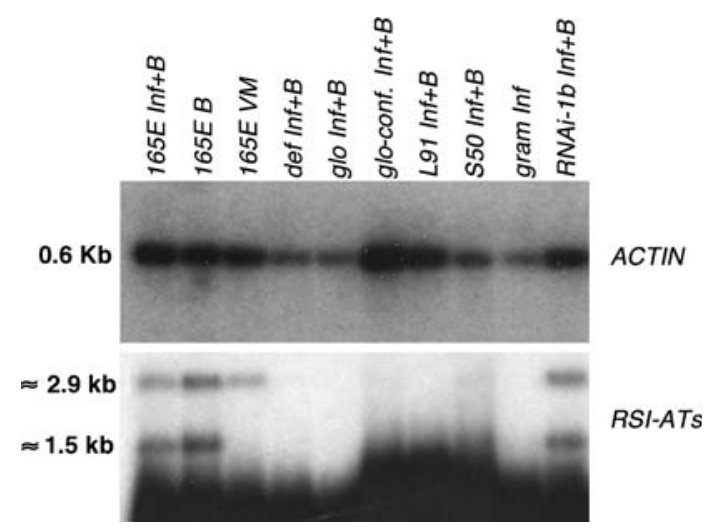

Fig. 4 Detection of additional RSI-ATs by RT-PCR. Plant material from different $A$. majus lines was used to extract mRNA. The TNP forward primer was derived from a predicted exon of this gene, while the reverse primer was designed from the RSI transcript. The ACTIN gene was used as a control to semi-quantify the amount of single-stranded DNA used in the PCR reaction. The amplified products and their sizes are indicated on the right and left side of the panel, respectively. Inf inflorescence, $B$ buds, $V M$ vegetative meristems. Plant lines: 165E; def: deficiens-globifera; glo: globosa; glo-conf.: globosa-confusa; L91: Line 91; S50: Sippe 50; gram: graminifolia RNAi-1b: a transgenic line carrying a construct to silence RSI (Roccaro et al. 2005). The smear at the bottom of the Southern blot is likely due to breakdown of the radioactively labelled first strand during PCR and/or linear amplification of breakdown products which have lost one primer-annealing site, but which still can hybridise to the radioactive probe 
Parts of RSI RNA and the TNP RNA form double-stranded RNA

The detection of the RSI-ATs suggested that doublestranded RNAs (dsRNA) might form in cells where both RSI and RSI-ATs transcripts are expressed. To detect dsRNA we took advantage of the methods described in Korneev et al. (1999), which exploited the feature of the RNase-ONE enzyme to degrade single-stranded RNA leaving dsRNA intact. Total RNA was extracted under non-denaturing conditions, treated with an excess of RNaseONE and reverse-transcribed with primers located outside and inside the hypothetical dsRNA molecules (Fig. 5). If dsRNA molecules existed, then a single product is detected from the sample treated with RNase-ONE and reverse transcribed with the primer inside the dsRNA molecule. In contrast, no product would be detected from the samples reverse transcribed with the outside primer. dsRNA could be detected in the inflorescence apices of the $165 \mathrm{E}$ line (Fig. 5, lane 8). The expected product of $322 \mathrm{bp}$ was cloned and sequenced confirming that it originated from $m R S I$. This finding possibly links the control of $R S I$ expression to an antisense type of gene regulation and the existence of dsRNA molecules to the production of siRNA.

TamRSI transcriptional levels in carpels

The complexity of the RSI transcriptional regulation was previously shown in Antirrhinum transgenic plants carrying a
RNA interfering (RNAi) construct designed to silence the RSI gene(s) (Roccaro et al. 2005). In these transgenic lines (RNAi- $1 a$ and $R N A i-1 b)$ a general up-regulation of the full length $m R S I$ with a concomitant down-regulation of the RSIATl transcript was observed, the latter monitored by the amount of a $329 \mathrm{bp} \mathrm{PCR} \mathrm{product} \mathrm{corresponding} \mathrm{to} \mathrm{the} 3^{\prime}$ end of the RSI-ATI transcript. This could be explained by a silencing effect exerted by the sense strand of the transgenic dsRNA exclusively on the RSI-ATI transcript. Reducing RSI-ATI transcript level appeared to favour an up-regulation of the $R S I$ mRNA. However, when we analysed the level of expression of RSI and RSI-ATI in the carpels of the RNAi-1 lines by quantitative RT-PCR, we surprisingly found that both transcripts were up-regulated compared to their level of expression in the carpels of the wild type line $165 E$ (Fig. 6a). Currently, it is not known which regulatory mechanism established this transcriptional effect in the transgenic lines, although we favour the idea that in carpels the up-regulation of full length $R S I$ mRNA and of the RSI-ATI RNA is the result of the activity of a carpel specific RNA-dependent RNA polymerase (RdRP) (Xie et al. 2004) induced by the transgenic silencing construct. Nevertheless, this alteration of the RSI and RSI-ATI transcript levels seemed to have developmental consequences for the seeds produced by the transgenic $R N A i-1$ lines.

Seed phenotype of the transgenic lines

In wild type carpels, $R S I$ is mainly expressed in the style and in the ovules (Roccaro et al. 2005) at a later stage of

A

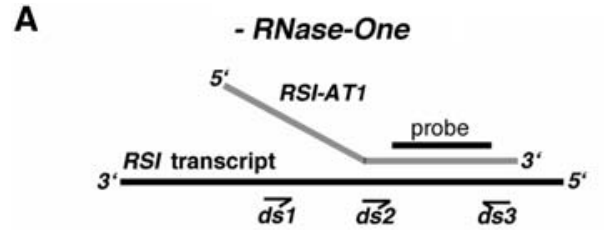

+ RNase-One
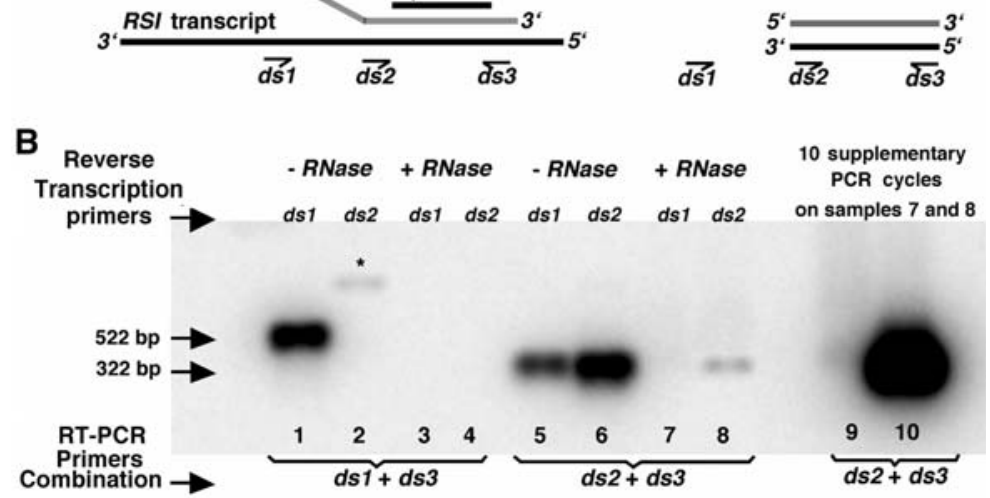

Fig. 5 Detection of dsRNA. a Schematic representation of doublestranded RNA formation. b PCR products generated after 15 cycles of amplification with the primer combination indicated and detected by Southern hybridization using the probe indicated in $\mathbf{a}$. The RNA sample extracted in non-denaturing condition from line $165 \mathrm{E}$ was divided into two fractions and treated with DNase to remove traces of DNA, and with DNase plus RNase-ONE to remove DNA and single-stranded RNA, respectively. The two resulting samples were reverse transcribed with primers ds 1 and ds 2 respectively, and subjected to PCR with the primer combination indicated. The $522 \mathrm{bp}$ product is derived from a
$R S I$ transcript, as expected from mRNA treated only with DNase and reverse transcribed with the primer ds 1 . Samples in lanes 2 and 4 were not expected to give a PCR product, because they did not contain a complementary ds 1 sequence due to the reverse transcription with the primer ds2. Similarly, for the samples in lanes 3 and 7 the treatment with RNase-ONE removed transcripts containing sequence complementary to ds1, therefore no product was amplified. The $322 \mathrm{bp}$ band represents the expected product generated from dsRNA molecules. The asterisk indicates an unspecific $P C R$ product 
Fig. 6 a Quantitative RT-PCR performed on inflorescences including floral buds (Inf. + Buds) and on carpels, to show the level of expression of the full length $R S I$ and of the 329 bp long RSI-AT1 transcripts in the lines $165 E$ and $R N A i-1$. b Seed phenotype of the self-pollinated lines $165 E(1-2)$ and RNAi-1a (3-4), and of the two reciprocal crosses (5-6 and 7-8, respectively) (see text for details); c chart of the seed weight. The same seed phenotype was obtained with the $R N A i-1 b$ line. Scale bar in b 1, 3, 5, 6 and 7, $2 \mathrm{~mm} ; 2,4$ and $8,500 \mu \mathrm{m}$
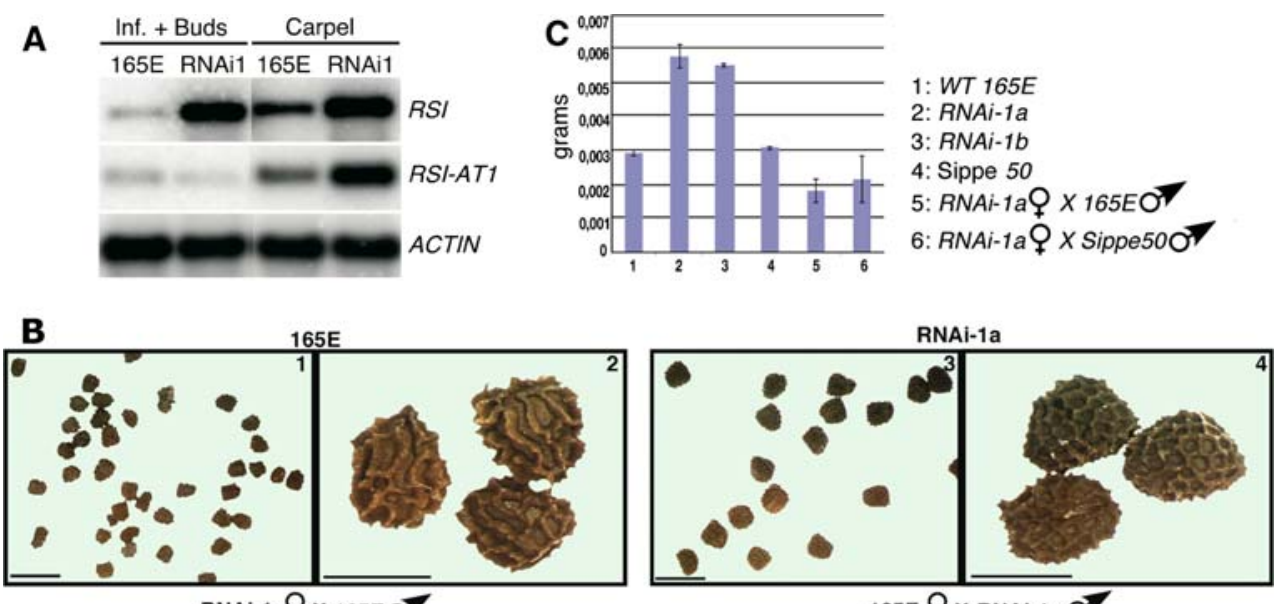

RNAi-1a? X 165E O
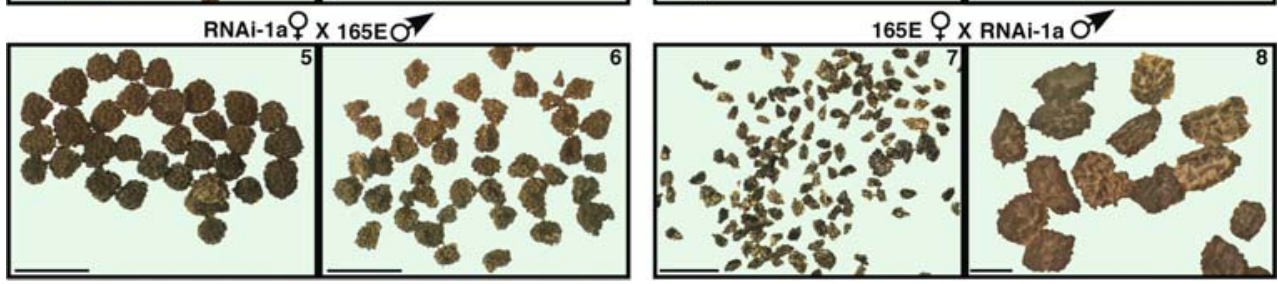

flower development. The seed capsules of the two RNAi-1 lines lack the four pores necessary for seed dispersal that are normally localised on the top of the seed capsule (Suppl. Fig. 6). The seed coat of seeds coming from selfpollinated $R N A i-1$ plants, and from those backcrossed with several wild type lines used as male gametophyte donors, had a different sculpture (pattern of ridges). In wild type lines, the seed coat had a discrete longitudinal pattern of ridges, in contrast to a more reticulate pattern of ridges seen in the RNAi-1 lines (Fig. 6, compare panel B2 with B4 and B5-6). In the reciprocal cross (RNAi- 1 lines as pollen donors), the seed coat had a wild type phenotype (Fig. 6, panel B7-8) in agreement with the maternal origin of the seed coat. Therefore, it appears that altered levels of expression of $R S I$ and $R S I-A T I$ transcripts in the female organ influence seed dispersal and the architecture of the protecting layer of A. majus seeds.

Self-pollinated transgenic flowers produced fewer but larger seeds weighting almost twice as much as those of the wild type lines (Fig. 6, panel B and C). Backcrosses to the wild type lines (pollen donor) yielded seeds half of which had the external dimensions like those of the self-pollinated RNAi-1 lines (Fig. 6, B5), while the other half had a "shrunken" phenotype (Fig. 6, B6). The non-shrunken seeds however weighed less than the wild type seeds (Fig. 6c) because most of them were without embryo and endosperm, though their seed coats were well developed. The seed population of the reciprocal backcrosses (RNAi-1 lines as pollen donors) resulted in seeds smaller then wild type with a few normal-sized seeds in a ratio of about 12:1 (Fig. 6, B7-8). Hence, the reciprocal crosses showed an evident parent-of-origin effect similar to mutations in imprinted genes (Spielman et al. 2001). A molecular and genetic explanation of the observed seed phenotypes will surely require more detailed studies. However, several scenarios can be envisaged to explain these dramatic effects on seed development, which are discussed below.

\section{Discussion}

$R S I$ represents a new type of CACTA transposable element

We have demonstrated by several means that the RSI gene is a constituent of a CACTA transposon in A. majus. Tam$R S I$ is present in multiple copies at non-allelic positions, suggesting that it is able to transpose within the Antirrhinum genome. This is corroborated by the analysis of different excision products obtained from the integration sites of TamRSI-2 (Fig. 3). A putative TRANSPOSASE (TNP) gene is found within the transposon unit in opposite orientation with respect to the RSI gene. This is an unusual structure not seen in other CACTA-type TEs. The first described transposon structure with multiple ORFs in a convergent orientation was the Mutator element of Zea mays (Hershberger et al. 1995). Recently a new class of complex DNA transposons called polintons has been reported (Kapitonov and Jurka 2006). This type of transposable element also contains multiple ORFs often arranged in convergent orientation. How could such a structure arise?

Studies in several plant species have described the acquisition of gene fragments by TEs including CACTA transposons (Fischer et al. 1995; Kawasaki and Nitasaka 2004), $M u D R$ and $M u D R$-like elements, (Talbert and Chandler 
1988; Juretic et al. 2005), Helitron elements (Morgante et al. 2005), as well as two cases of LTR-retrotransposons in maize (Bureau et al. 1994; Jin and Bennetzen 1994). The mechanism of gene fragment incorporation, termed "transduplication", which preserves the exon-intron structure of the gene fragment acquired, is unknown (Juretic et al. 2005). In contrast to the transduplicated gene fragments found in rice, in Arabidopsis and in the Japanese morning glory that consist mostly of non-expressed genes (Kawasaki and Nitasaka 2004; Hoen et al. 2006), RSI expression is developmentally regulated (Roccaro et al. 2005), and it codes for an expressed protein (M. Roccaro, unpublished ). Three cases of transposase-derived genes have been identified recently in plants, indicating that transposons can be beneficial for their own host (Hudson et al. 2003; Bundock and Hooykaas 2005; Muehlbauer et al. 2006).

It can be speculated that, in the case of RSI, two independent insertions of a CACTA transposon occurred at the $5^{\prime}$ and $3^{\prime}$ end of an ancient $R S I$ gene, containing a DNA binding domain and/or a dimerization domain. The $3^{\prime}$ end insertion occurred in the opposite orientation to the transcription of the ancient RSI. Subsequently, a number of simultaneous or independent rearrangement steps of the two CACTA transposons may have produced a new transposon unit, consisting of $R S I$ and TNP embedded between the CACTA TIRs. Alternatively, the RSI gene could result from rearrangements of a tnpA-like gene within the CACTA transposon unit to form a structure like the ones described above. Although these are speculative views, it is interesting to note that the transposase portion is relatively well conserved among the CACTA TE family, including the TNP of TamRSI (data not shown). In contrast, the TNPA part shows a high degree of sequence divergence as reported for the TNPA of En/Spm and Taml (Kunze and Weil 2002). Similarly, in a TamRSI-like structure present in the Antirrhinum genome (Causier et al. 2005, Suppl. Fig. 1, CACTA TIRs non-annotated), the RSI-like part shows only a weak similarity with other proteins, including RSI itself (data not shown), whereas the TNP portion is more conserved. Therefore, it appears that the "TNPA/RSI"-like genes are faster evolving portions of the CACTA transposons, and one way to achieve this variability of sequence could be through the acquisition of gene fragment(s).

RSI was first isolated because of its ability to bind a specific region of the DEFICIENS (DEF) promoter and to modulate the activity of $D E F$ during petal and stamen development. Transcriptional repression and activation were two suggested functions of RSI (Roccaro et al. 2005). The finding that RSI is a constituent of a CACTA transposon, together with its trans-acting regulatory activity exerted on the $D E F$ gene could justify to call TamRSI a "controlling element".
dsRNA and $R S I$ transcriptional regulation

Overlapping antisense transcripts have the potential to form dsRNAs that in turn can form small RNA molecules called short interfering RNAs (siRNAs), which mediate in a sequence-specific manner post-transcriptional gene silencing (PTGS) in plants (Hamilton and Baulcombe 1999), and in a sequence- and strand-specific manner RNA interference (RNAi) in animals (Fire et al. 1998; Zamore et al. 2000; Schwarz et al. 2003,), as well as transcriptional gene silencing (TGS) (Matzke et al. 2001). SiRNA precursors are also formed by the activity of RNA-dependent RNA polymerases (RdRP) (Xie et al. 2004). The detection of RSI-ATs transcripts, most likely formed by internal deletion-fusion events of TamRSI, and the identification of a dsRNA formed by annealing of these aberrant transcripts with the sense RSI RNA, suggested that RSI and TNP expression are under the control of PTGS and/or TGS regulatory mechanisms. A number of identified siRNAs have been related to transposon sequences in plants and animals (Llave et al. 2002; Mette et al. 2002; Aravin et al. 2003; Sijen and Plasterk 2003), and siRNAs have also been shown to regulate plant transposon activity, assembly of heterochromatin and DNA methylation in transposon rich regions such as centromers (Lippman et al. 2004). A selfenforcing RNAi loop mechanism, involving tandem repeats, the activity of an RdRP and the cyclic production of siRNAs, has been described in $S$. pombe (Volpe et al. 2002; Martienssen 2003) to account for the silencing of transposons in heterochromatic regions. Such a model could in part explain the higher transcriptional level of $R S I$ and $R S I-A T I$ detected in the carpels of the transgenic RNAi1 lines. In carpels (ovules) the RNAi construct, intended to silence RSI, produces dsRNA molecules that, once processed into siRNAs, could serve as primers to maintain the synthesis of new dsRNA precursors by a carpel specific RdRP (Fig. 7). These precursors are processed to siRNAs, which in turn regulate RSI and TNP expression of TamRSI. The identification of siRNAs containing RSI/TNP sequences would be instrumental to further explore the transcriptional mechanism regulating $R S I$ expression.

\section{Seed phenotypes}

The observed seed phenotype of the two RSI RNAi- 1 lines is a very intriguing aspect of TamRSI function. Reciprocal crosses performed with the RNAi-l lines strongly suggested that the imprinting effect is somehow caused either by the up-regulation of $R S I$ transcript in the stamen (Roccaro et al. 2005 ) or by $R S I$ and $R S I-A T I$ transcripts in the carpels. Since PTGS is a highly sequence-specific mechanism, another mechanism must be invoked to explain the seed phenotype. An intriguing question is whether the observed 


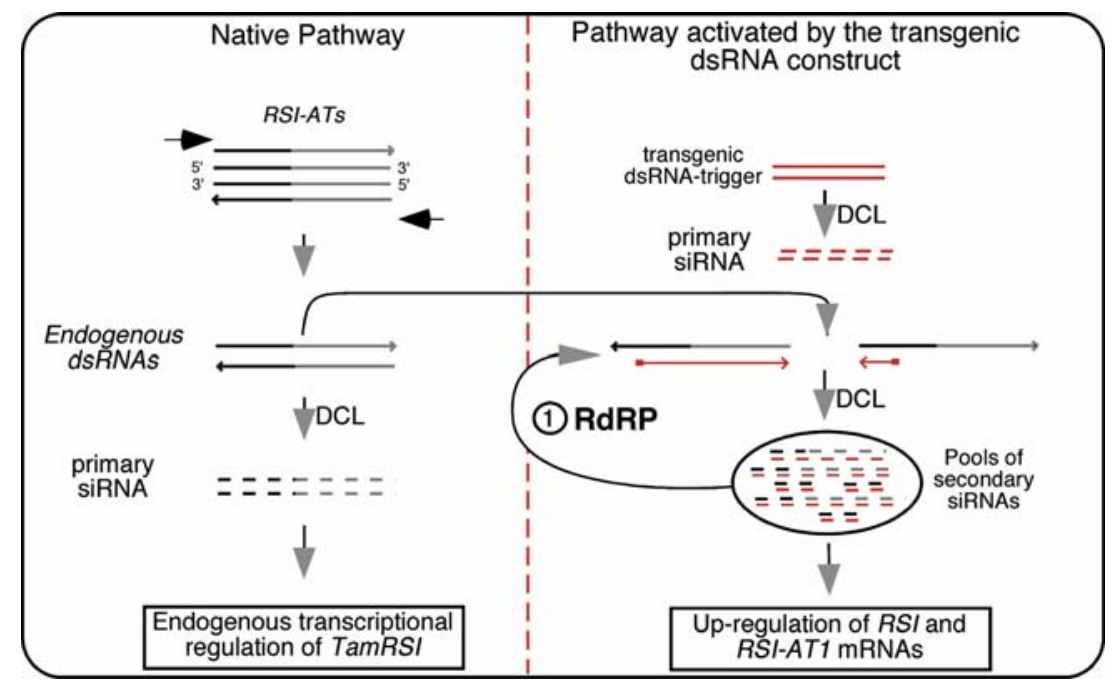

Fig. 7 Proposed model for the transcriptional regulation of TamRSI in the native and the transgenic pathways. Native and transgenic $d s R N A$ precursors are processed in siRNAs by the activity of a plant Dicer-like (DCL) protein (Schauer et al. 2002), generating pools of siRNA molecules. The native pathway establishes a complex balance between the levels of expression of RSI, TNP (not indicated) and RSI-ATs transcripts and the level of $s i R N A s$ produced from them in stamen and carpels. In carpels however, a self-enforcing loop (indicated by 1 ) is envisaged in

seed phenotype is due to RSI activity as transcription factor, or it reflects an epigenetic effect caused by $R S I$ as a part of a transposon. A link between transposons and silencing of imprinted genes has been recently discovered for the imprinted gene FWA. A short interspersed nucleotide element (SINE) located in the promoter region of $F W A$ is responsible for the silencing of this gene (Martienssen et al. 2004; Kinoshita et al. 2007). The silencing effect is associated with the production of siRNA, with DNA methylation and histone methylation (Soppe et al. 2000; Chan et al. 2004; Lippman et al. 2004). However, another well-characterised imprinted gene, MEDEA, appears to be regulated by a mechanism that does not involve transposons, though methylation is still required (Xiao et al. 2003; Spillane et al. 2004). It can be speculated that the up-regulation of $R S I$ and/ or RSI-ATI transcripts in the carpels of the transgenic lines might lead to a methylation, guided by the over production of siRNAs, of maternally inherited genes involved in the seed development, which might be located near TamRSI copies. This hypothetical methylation effect on the maternal genes could explain the paternalized phenotype observed (big seed, failure in endosperm/embryo development) in the cross where the ovule donors are the transgenic lines. Similarly, in the reciprocal cross (pollen donor the RNAi-1 lines) the maternalized (small seed) phenotype could be the result of methylation of paternally inherited genes. However, since hyper- or hypo-methylation can have the same effect on seed development, depending on whether the maternal or the paternal chromosome undergoes these two opposite types of the pathway triggered by the transgenic $d s R N A$ and mediated by the activity of a carpel specific $R d R P$. The production of secondary siRNAs could, in turn, feed the loop and alter the balance of endogenous siRNAs resulting in an up-regulation of the TamRSI transcripts. The black filled arrows indicate the direction of transcription. The black and grey parts of aberrant $R S I$ transcripts represent an $R S I$ and the $T N P$ genes or fragments of them, respectively, in a convergent configuration and generated by an internal deletion-fusion similar to that of TamRSI-3

methylation (Spielman et al. 2001), more than one scenario is possible. For instance, the down-regulation of the RSIATl transcript in the transgenic stamen (pollen) (Roccaro et al. 2005) would lead to a hypo-methylation of maternal genes on the paternal chromosomes with a consequently maternalized seed phenotype. An alternative explanation is that the up-regulation of $R S I$ in stamen and carpels directs the activation or repression of genes that are normally imprinted in the maternal or paternal genomes, respectively. This latter view would favour the idea of RSI as a TNPAlike protein able to exert a de-methylation/activation function as previously proposed for the En/Spm tnpA gene (Fedoroff et al. 1995). More extensive studies are required to unravel the functions of this unique CACTA transposon and to clarify the involvement of $R S I$ in floral development as well as in seed development.

Acknowledgments We would like to thank Z. Schwarz-Sommer for the inbred lines investigated in this project and for the A. majus X A. molle F2 DNA samples to map some of the TamRSI copies. We also thank T. Colby, C. Micali and M. Humphry for editing the manuscript. M.R was supported by the SFB project 572 ; Y. L. was supported by a Max-Planck Fellowship.

\section{References}

Alleman M, Sidorenko L, McGinnis K, Seshadri V, Dorweiler JE, White J, Sikkink K, Chandler VL (2006) An RNA-dependent RNA polymerase is required for paramutation in maize. Nature 442:295-298 
Aravin AA, Lagos-Quintana M, Yalcin A, Zavolan M, Marks D, Snyder B, Gaasterland T, Meyer J, Tuschl T (2003) The small RNA profile during Drosophila melanogaster development. Dev Cell 5:337-350

Bonas U, Sommer H, Saedler H (1984) The 17-Kb Tam-1 element of Antirrhinum majus induces a 3-bp duplication upon integration into the Chalcone Synthase gene. EMBO J 3:1015-1019

Bundock P, Hooykaas P (2005) An Arabidopsis hAT-like transposase is essential for plant development. Nature 436:282-284

Bureau TE, White SE, Wessler SR (1994) Transduction of a cellular gene by a plant retroelement. Cell 77:479-480

Causier B, Castillo R, Zhou JL, Ingram R, Xue YB, Schwarz-Sommer Z, Davies B (2005) Evolution in action: following function in duplicated floral homeotic genes. Curr Biol 15:1508-1512

Chan SWL, Zilberman D, Xie ZX, Johansen LK, Carrington JC, Jacobsen SE (2004) RNA silencing genes control de novo DNA methylation. Science 303:1336-1336

Coen ES, Carpenter R, Martin C (1986) Transposable elements generate novel spatial patterns of gene-expression in Antirrhinum majus. Cell 47:285-296

Doolittle WF, Sapienza C (1980) Selfish genes, the phenotype paradigm and genome evolution. Nature 284:601-603

Fedoroff N, Schläppi M, Raina R (1995) Epigenetic regulation of the maize Spm transposon. Bioessays 14:291-297

Fire A, Xu S, Montgomery MK, Kostas SA, Driver SE, Mello CC (1998) Potent and specific genetic interference by double-stranded RNA in Caenorhabditis elegans. Nature 391:806-811

Fischer A, Baum N, Saedler H, Theissen G (1995) Chromosomal mapping of the MADS-Box multigene family in Zea mays reveals dispersed distribution of allelic genes as well as transposed copies. Nucleic Acids Res 23:1901-1911

Gierl A, Lütticke S, Saedler H (1988) TnpA product encoded by the transposable element En-1 of Zea mays is a DNA binding protein. EMBO J 7:4045-4053

Hamilton AJ, Baulcombe DC (1999) A species of small antisense RNA in posttranscriptional gene silencing in plants. Science 286:950 952

Hershberger RJ, Benito MI, Hardeman KJ, Warren C, Chandler VL, Walbot V (1995) Characterization of the major transcripts encoded by the regulatory MuDR transposable element of maize. Genetics 140:1087-1098

Hoen DR, Park KC, Elrouby N, Yu ZH, Mohabir N, Cowan RK, Bureau TE (2006) Transposon-mediated expansion and diversification of a family of ULP-like genes. Mol Biol Evol 23:12541268

Hudson ME, Lisch DR, Quail PH (2003) The FHY3 and FAR1 genes encode transposase-related proteins involved in regulation of gene expression by the phytochrome A-signaling pathway. Plant J 34:453-471

Jacob F, Monod J (1961) Genetic regulatory mechanisms in synthesis of proteins. J Mol Biol 3:318-356

Jin YK, Bennetzen JL (1994) Integration and nonrandom mutation of a plasma-membrane proton ATPase gene fragment within the Bs1 retroelement of maize. Plant Cell 6:1177-1186

Juretic N, Hoen DR, Huynh ML, Harrison PM, Bureau TE (2005) The evolutionary fate of MULE-mediated duplications of host gene fragments in rice. Genome Res 15:1292-1297

Kapitonov VV, Jurka J (2006) Self-synthesizing DNA transposons in eukaryotes. Proc Natl Acad Sci USA 103:4540-4545

Kawasaki S, Nitasaka E (2004) Characterization of Tpn1 family in the Japanese morning glory: En/Spm-related transposable elements capturing host genes. Plant Cell Physiol 45:933-944

Ketting RF, Haverkamp THA, van Luenen H, Plasterk RHA (1999) mut-7 of C. elegans, required for transposon silencing and RNA interference, is a homolog of Werner syndrome helicase and RNaseD. Cell 99:133-141
Kinoshita Y, Saze H, Kinoshita T, Miura A, Soppe WJ, Koornneef M, Kakutani T (2007) Control of FWA gene silencing in Arabidopsis thaliana by SINE-related direct repeats. Plant J 49:38-45

Korneev SA, Park JH, O’Shea M (1999) Neuronal expression of neural nitric oxide synthase (nNOS) protein is suppressed by an antisense RNA transcribed from an NOS pseudogene. J Neurosci 19:7711-7720

Kunze R, Weil CF (2002) The hAT and CACTA superfamilies of plant transposon. In: Craig NL, Craigie R, Gellert M, Lambowitz (eds) Mobile DNA II ASM Press, Washington DC, pp 565-610

Lippman Z, Gendrel AV, Black M, Vaughn MW, Dedhia N, McCombie WR, Lavine K, Mittal V, May B, Kasschau KD, Carrington JC, Doerge RW, Colot V, Martienssen R (2004) Role of transposable elements in heterochromatin and epigenetic control. Nature 430:471-476

Llave C, Kasschau KD, Rector MA, Carrington JC (2002) Endogenous and silencing-associated small RNAs in plants. Plant Cell 14:1605-1619

Martienssen RA (2003) Maintenance of heterochromatin by RNA interference of tandem repeats. Nat Genet 35:213-214

Martienssen R, Lippman Z, May B, Ronemus M, Vaughn M (2004) Transposons, tandem repeats, and the silencing of imprinted genes. Cold Spring Harb Symp Quant Biol 69:371-379

Masson P, Rutherford G, Banks JA, Fedoroff N (1989) Essential large transcripts of the maize Spm transposable element are generated by alternative splicing. Cell 58:755-765

Matzke M, Matzke AJ, Kooter JM (2001) RNA: guiding gene silencing. Science 293:1080-1083

McClintock B (1950) The origin and behavior of mutable loci in maize. Proc Natl Acad Sci USA 36:344-355

McClintock B (1956) Controlling elements and the gene. Cold Spring Harb Symp Quant Biol 16:197-216

McClintock B (1965) The control of gene action in maize. Brookhaven Symp Biol 18:162-184

Mette MF, van der Winden J, Matzke M, Matzke AJM (2002) Short RNAs can identify new candidate transposable element families in Arabidopsis. Plant Physiol 130:6-9

Morgante M, Brunner S, Pea G, Fengler K, Zuccolo A, Rafalski A (2005) Gene duplication and exon shuffling by helitron-like transposons generate intraspecies diversity in maize. Nat Genet 37:997-1002

Muehlbauer GJ, Bhau BS, Syed NH, Heinen S, Cho SH, Marshall D, Pateyron S, Buisine N, Chalhoub B, Flavell AJ (2006) A hAT superfamily transposase recruited by the cereal grass genome. Mol Gen Genomics 275:553-563

Nevers P, Saedler H (1977) Transposable Genetic Elements as Agents of Gene Instability and Chromosomal Rearrangements. Nature 268:109-115

Pereira A, Cuypers H, Gierl A, Schwarz-Sommer Z, Saedler H (1986) Molecular analysis of the En/Spm transposable element system of Zea mays. EMBO J 5:835-841

Roccaro M, Li Y, Masiero S, Saedler H, Sommer H (2005) ROSINA (RSI), a novel protein with DNA-binding capacity, acts during floral organ development in Antirrhinum majus. Plant J 43:238-250

Sambrook J, Fritsch EF, Maniatis T (1989) Molecular cloning: a laboratory manual. Cold spring Laboratory Press, New York

Schauer SE, Jacobsen SE, Meinke DW, Ray A (2002) DICER-LIKE1: blind men and elephants in Arabidopsis development. Trends Plant Sci 7:487-491

Schiefelbein JW, Raboy V, Kim HY, Nelson OE (1988) Molecular characterization of Suppressor-mutator (Spm)-induced mutations at the bronze-1 locus in maize: the bz-m13 alleles. In: Nelson O (ed) Plenum Press, New York

Schwarz-Sommer Z, Gierl A, Klösgen RB, Wienand U, Peterson PA, Saedler H (1984) The Spm (En) transposable element controls the excision of a 2-kb DNA insert at the wx-m8 allele of Zea mays. EMBO J 3:1021-1028 
Schwarz DS, Hutvagner G, Du T, Xu ZS, Aronin N, Zamore PD (2003) Asymmetry in the assembly of the RNAi enzyme complex. Cell 115:199-208

Schwarz-Sommer Z, Silva ED, Berndtgen R, Lonnig WE Muller A, Nindl I, Stuber K, Wunder J, Saedler H, Gubitz T, Borking A, Golz JF, Ritter E, Hudson A (2003) A linkage map of an F-2 hybrid population of Antirrhinum majus and A. molle. Genetics 163:699-710

Sijen T, Plasterk RHA (2003) Transposon silencing in the Caenorhabditis elegans germ line by natural RNAi. Nature 426:310-314

Sommer H, Bonas U, Saedler H (1988) Transposon-induced alterations in the promoter region affect transcription of the chalcone synthase gene of Antirrhinum majus. Mol Gen Genet 211:49-55

Soppe WJJ, Jacobsen SE, Alonso-Blanco C, Jackson JP, Kakutani T, Koornneef M, Peeters AJM (2000) The late flowering phenotype of fwa mutants is caused by gain-of-function epigenetic alleles of a homeodomain gene. Mol Cell 6:791-802

Spielman M, Vinkenoog R, Dickinson HG, Scott RJ (2001) The epigenetic basis of gender in flowering plants and mammals. Trends Genet 17:705-711

Spillane C, Baroux C, Escobar-Restrepo JM, Page DR, Laoueille S, Grossniklaus U (2004) Transposons and tandem repeats are not involved in the control of genomic imprinting at the MEDEA locus in Arabidopsis. Cold Spring Harb Symp Quant Biol 69:465-475

Tabara H, Sarkissian M, Kelly WG, Fleenor J, Grishok A, Timmons L, Fire A, Mello CC (1999) The rde-1 gene, RNA interference, and transposon silencing in C elegans. Cell 99:123-132
Talbert LE, Chandler VL (1988) Characterization of a highly conserved sequence related to Mutator transposable elements in maize. Mol Biol Evol 5:519-529

Tijsterman M, Ketting RF, Plasterk RHA (2002) The genetics of RNA silencing. Annu Rev Genet 36:489-519

Trentmann SM, Saedler H, Gierl A (1993) The transposable element En/Spm-encoded TNPA proteins contains a DNA binding and a dimerization domain. Mol Gen Genet 238:201-208

Volpe TA, Kidner C, Hall IM, Teng G, Grewal SIS, Martienssen RA (2002) Regulation of heterochromatic silencing and histone H3 lysine-9 methylation by RNAi. Science 297:1833-1837

Wilkinson M, Silva ED, Zachgo S, Saedler H Schwarz-Sommer Z (2000) CHORIPETALA and DESPENTEADO: general regulators during plant development and potential floral targets of FIMBRIATA-mediated degradation. Development 127:3725-3734

Xiao WY, Gehring M, Choi Y, Margossian L, Pu H, Harada JJ, Goldberg RB, Pennell RI, Fischer RL (2003) Imprinting of the MEA polycomb gene is controlled by antagonism between MET1 methyltransferase and DME glycosylase. Dev Cell 5:891-901

Xie ZX, Johansen LK, Gustafson AM, Kasschau KD, Lellis AD, Zilberman D, Jacobsen SE, Carrington JC (2004) Genetic and functional diversification of small RNA pathways in plants. PLoS Biol 2:642-652

Zamore PD, Tuschl T, Sharp PA, Bartel DP (2000) RNAi: Doublestranded RNA directs the ATP-dependent cleavage of mRNA at 21 to 23 nucleotide intervals. Cell 101:25-33 